\title{
Tracheal Obstruction
}

National Cancer Institute

\section{Source}

National Cancer Institute. Tracheal Obstruction. NCI Thesaurus. Code C78645.

Blockage of the lumen of the trachea. Causes include secretions, foreign bodies, and tumors. 